\title{
LATTICE-INVARIANT PROPERTIES OF TOPOLOGICAL SPACES
}

\author{
YIM-MING WONG
}

\begin{abstract}
W. J. Thron proved in 1962 that regularity and normality are lattice-invariant properties but not $T_{0}$ and $T_{1}$. In the present paper it is proved that complete regularity, compactness, local compactness, Lindelöf, second countability and connectedness are lattice-invariant properties. It is also proved that Hausdorff, complete normality, separability, and first countability are not lattice-invariant properties.
\end{abstract}

1. Two topological spaces are said to be lattice-equivalent if their lattices of open sets are isomorphic. It follows by duality that this is the case iff their lattices of closed sets are isomorphic. A topological property $P$ is said to be lattice-invariant if a topological space latticeequivalent to a topological space having $P$ has also $P$.

W. J. Thron, in his paper [1], treated among others the lattice characterization of some separation axioms for topological spaces. He proved that $T_{0}$ and $T_{1}$ are not lattice-invariant. He also proved that regularity and normality are lattice-invariant.

In this paper, we shall prove that complete regularity, compactness, local compactness (in a sense), Lindelöf, second countability, and connectedness are lattice-invariant properties. We also prove that Hausdorff, complete normality, separability, and first countability are not lattice-invariant properties.

2. Let $X$ be a topological space and $\mathscr{L}$ the lattice of all open sets in $X$. The following results are evident.

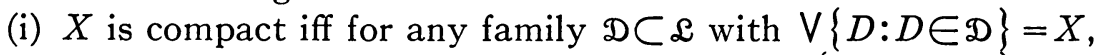
there is a finite subfamily $D_{0}$ of $D$ such that $\vee\left\{D: D \in D_{0}\right\}=X$.

(ii) $X$ is second countable iff there is a countable family $D \subset \mathscr{L}$ such that any element in $\mathscr{L}$ is a lattice sum of some elements in $\mathscr{D}$.

(iii) $X$ is Lindelöf iff for any family $D \subset \mathscr{L}$ with $\vee\{D: D \in D\}=X$, there is a countable subfamily $D_{0}$ of $D$ such that $\vee\left\{D: D \in D_{0}\right\}=X$.

(iv) $X$ is connected iff $X$ is not a lattice sum of two disjoint nonzero elements in $\mathscr{L}$.

Received by the editors December 8, 1969.

A MS 1969 subject classifications. Primary 5420; Secondary 0630, 5423, 5440, 5452, 5455.

Key words and phrases. Lattice-invariant property, complete regularity, compactness, local compactness, Lindelöf, second countability, connectedness, Hausdorff, complete normality, separability, first countability. 
Using the above lattice characterizations, we derive the following theorem.

THEOREM 1. Compactness, second countability, Lindelöf and connectedness are lattice-invariant properties.

3. Let $X$ be a topological space. According to E. F. Steiner's definition, a family $\mathcal{C}$ of closed sets in $X$ is said to be separating if for every closed set $F$ in $X$ and $x \in X \backslash F$, there exist $C_{1}, C_{2} \in \mathcal{C}$ such that $F \subset C_{1}, x \in C_{2}$ and $C_{1} \cap C_{2}=\varnothing$. $\mathrm{e}$ is said to be normal if for every $A, B \in \mathcal{e}$ with $A \wedge B=\varnothing$, there exist $C, D \in \mathcal{e}$ such that $C \cap B=\varnothing$, $A \cap D=\varnothing$ and $C \cup D=X$. In his paper [2], E. F. Steiner proved that a topological space is completely regular iff it possesses a normal separating family of closed sets.

Let $\&$ be the lattice of all closed sets in $X$. Making use of the above statement, we shall prove that $X$ is completely regular iff there is a subset $\mathfrak{C}$ of $\&$ such that the following two conditions are satisfied.

(i) For every $F \in \mathscr{L}$ such that $F \neq X$, there exist sets $\left\{D_{i}: i \in I\right\} \subset \mathbb{e}$ and $\left\{E_{i}: i \in I\right\} \subset \mathcal{e}$ such that $F=\Lambda\left\{D_{i}: i \in I\right\}, E_{i} \bigvee D_{i}=X$ and $E_{i} \wedge F=\varnothing$ for all $i \in I$.

(ii) For given $A, B \in \mathfrak{C}$, with $A \wedge B=\varnothing$, there exist $D, E \in \mathfrak{e}$ such that $A \wedge D=\varnothing, B \wedge E=\varnothing$ and $D \vee E=X$.

Proof. Necessity. Let $X$ be a completely regular space and let $\mathcal{e}$ be a normal separating family of closed sets. We shall prove that $\mathcal{e}$ satisfies (i) and (ii).

(i) For $F \in \mathcal{L}, F \neq X$ and any point $x \in X \backslash F$, there exist $A_{x}$, $B_{x} \in \mathcal{C}$ such that $A_{x} \wedge B_{x}=\varnothing$ and $x \in A_{x}, B_{x} \supset F$. By the normality of $\mathfrak{e}$, there are $E_{x}, D_{x} \in \mathcal{C}$ such that $E_{x} \wedge B_{x}=\varnothing, D_{x} \wedge A_{x}=\varnothing$ and $E_{x} \bigvee D_{x}=X$.

We shall prove that the sets $\left\{D_{x}: x \in X \backslash F\right\}$ and $\left\{E_{x}: x \in X \backslash F\right\}$ satisfy the requirements. Now $E_{x} \bigvee D_{x}=X$ for all $x \in X \backslash F$ follows from definition, and $E_{x} \wedge F=\varnothing$ for all $x \in X \backslash F$ follows from $E_{x} \wedge B_{x}$ $=\varnothing$ and $B_{x} \supset F$. We now prove $F=\Lambda\left\{D_{x}: x \in X \backslash F\right\}$. If $x \notin F$, then $x \in A_{x}$ and hence $x \notin D_{x}$. Therefore $x \notin \Lambda\left\{D_{x}: x \in X \backslash F\right\}$. Conversely if $t \notin \wedge\left\{D_{x}: x \in X \backslash F\right\}$, then $t \notin D_{x}$ for some $x \notin F$. But $D_{x} \bigvee E_{x}=X$ and $E_{x} \wedge B_{x}=\varnothing$, we get $t \notin B_{x}$ for some $x \notin F$. Now $B_{x} \supset F$ therefore $x \notin F$ and hence $F=\Lambda\left\{D_{x}: x \in X \backslash F\right\}$.

(ii) The normality of $\mathfrak{e}$ follows immediately.

Sufficiency. Let $\mathfrak{e}$ be the set of closed sets satisfying (i) and (ii).

Clearly, (ii) implies the normality of $\mathfrak{e}$. To prove $\mathfrak{C}$ is separating, let $x \in X \backslash F$ for some closed set $F$ in $X$. By (i), $F$ is the intersection of elements of $\mathfrak{e}$, there we can find $C \in \mathfrak{e}$ such that $x \notin C$ and $C \supset F$. Apply (i) again to the closed set $C \neq X$, we get $\left\{D_{i}: i \in I\right\} \subset \mathfrak{e}$ and 
$\left\{E_{i}: i \in I\right\} \subset \mathcal{e}$ satisfying requirement of (i). Now $x \notin C=\wedge\left\{D_{i}: i \in I\right\}$, therefore $x \notin D_{i}$ for some $i \in I$. It follows from $E_{i} \bigvee D_{i}=X$ that $x \in E_{i}$ for some $i \in I$. Therefore we get $E_{i}$ and $C$ of $\mathfrak{e}$ such that $x \in E_{i}$, $C \supset F$ and $E_{i} \cap C=\varnothing . \mathcal{e}$ is then separating.

From this characterization, we easily obtain the following.

TheOREM 2. Complete regularity is lattice-invariant.

4. A topological space is said to be locally compact if every point in the space has a neighborhood with compact closure.

Let $\mathscr{L}$ be the lattice of all open sets in a topological space $X$. For $A \in \mathscr{L}$, let $A^{\prime}$ be the largest element in $\mathscr{L}$ such that $A \wedge A^{\prime}=\varnothing . A$ is said to be a $P C$-element if for $D \subset \mathscr{L}$ such that $A^{\prime} \vee(\vee\{D: D \in D\})$ $=X$, there exists a finite subset $D_{0}$ of $D$ such that $A^{\prime} \vee\left(\vee\left\{D: D \in D_{0}\right\}\right)$ $=X$. It is evident that $X$ is locally compact iff $X=\mathrm{V}\{D: D \in D\}$ where $D$ is the family of all $P C$-elements of $\mathscr{L}$.

From the above characterization, we have the following.

THEOREM 3. Local compactness is lattice-invariant.

5. Let $X$ be a topological space consisting of only one point. $X$ is lattice-equivalent to any indiscrete space. Therefore, Hausdorff is not a lattice-invariant property.

Let $X$ be a discrete space containing uncountably many points. $X$ is clearly completely normal and not separable. Let $Y=X \cup\{z\}$, where $z$ is an element not in $X$. Let $\sigma=\{A \subset Y: z \in A\}$. Then $\sigma$ and $2^{X}$ are isomorphic. But $(Y, \sigma)$ is separable and is not normal hence not completely normal. This shows that complete normality and separability are not lattice-invariant properties.

Let $X$ be the set of the ordinals less than the first uncountable ordinal number $\Omega$. With the natural ordering, we define a topology for $X$ in the following manner. Let $M_{x}$ be the set consisting of all elements greater than $x$. The family $\tau$ consisting of $X, \varnothing$ and all $M_{x}$ forms a topology for $X$. It is easy to verify that $(X, \tau)$ is first countable. Let $Y=X \cup\{\Omega\}$, with a topology $\sigma$ defined similarly. It is clear that $(Y, \sigma)$ is not first countable. But $\tau$ and $\sigma$ are isomorphic. This shows that first countability is not lattice-invariant.

\section{REFERENCES}

1. W. J. Thron, Lattice-equivalence of topological spaces, Duke Math. J. 29 (1962), 671-679. MR 26 \#4307.

2. E. F. Steiner, Normal families and completely regular spaces, Duke Math. J. 33 (1966), 743-745. MR 33 \#7975.

University of Hong Kong, Hong Kong 